\title{
A Vanishing Theorem for Supersymmetric Quantum Field Theory and Finite Size Effects in Multiphase Cluster Expansions
}

\author{
Steven A. Janowsky ${ }^{1, \star}$ and Jonathan Weitsman ${ }^{2, \star \star}$ \\ 1 Department of Mathematics, Rutgers University, New Brunswick, NJ 08903, USA \\ 2 Department of Mathematics, University of California, Berkeley, CA 94720, USA
}

Received January 30, 1991; in revised form June 5, 1991

\begin{abstract}
We apply cluster expansion methods to the $N=2$ Wess-Zumino models in finite volume, in two space-time dimensions. We show that in the region of convergence of the cluster expansion, a vanishing theorem holds for the supercharge of the theory; that is, the dimension of the kernel of the Hamiltonian is equal to the index of the supercharge.
\end{abstract}

\section{Introduction}

In [8] a class of supersymmetric quantum field models in two spacetime dimensions were introduced and studied in finite volume. The methods of [8] were refined and extended in [9] by cluster expansion techniques which allowed us to obtain results in the infinite volume limit. In this paper we turn these methods back on the finite volume problem. We will find that they allow us to prove some substantial extensions of the results of [8].

We begin with a brief reprise of the results of $[8,9]$. Each two-dimensional $N=2$ Wess-Zumino model associates to a polynomial $V(z)$ (called the superpotential) a quantum field theory, consisting of a selfadjoint Hamiltonian $H$ densely defined on the Hilbert space $\mathscr{H}=\mathscr{H}_{b} \otimes \mathscr{H}_{f}$ corresponding to one massive complex (Dirac) Fermion field of mass $m$ and one complex Boson field with the same mass as the Fermion, defined on a circle $T_{l}$ of length $l$. Let $\Gamma=(-1)^{N_{f}}$ denote the Fermion parity operator. The supersymmetry of the Wess-Zumino model is expressed in the existence of a selfadjoint Fredholm operator $Q$ which satisfies the

\footnotetext{
* Supported in part by National Science Foundation Mathematical Sciences Postdoctoral Research Fellowship DMS 90-07206

$\star \star$ Supported in part by National Science Foundation Mathematical Sciences Postdoctoral Research Fellowship DMS 88-07291
} 
following relations:

$$
\begin{gathered}
Q^{2}=H, \\
Q \Gamma+\Gamma Q=0, \quad \Gamma^{2}=1 .
\end{gathered}
$$

The results of [8] may be summarized in the following theorem:

Theorem 1 [8]. The operator $e^{-\tau H}$ is trace class for all $\tau>0$; the index of the operator $Q$ is given by the formula

$$
\operatorname{ind}(Q)=\operatorname{Tr} \Gamma e^{-\tau H}=(\operatorname{deg} V)-1 .
$$

As an immediate corollary of this theorem and (1.1) we have the following:

Corollary 1.1. The dimension of the kernel of $H$ satisfies

$$
\infty>\operatorname{dim} \operatorname{ker} H \geqq(\operatorname{deg} V)-1 .
$$

We will consider the Wess-Zumino models corresponding to polynomials $V$ of the form

$$
\lambda^{-2} \tilde{W}(\lambda x)+\lambda^{-1} \xi w(\lambda x),
$$

where $\tilde{W}, w$ are polynomials of degree $n, \tilde{W}^{\prime}$ has $n-1$ distinct zeroes, and $\left|\tilde{W}^{\prime \prime}\right|=1$ at each such zero.

Remark. These conditions on the polynomial are basically those under which the cluster expansion methods of [9] apply.

The purpose of this paper is to prove the following theorem:

Theorem 2. For $\lambda$ and $\xi$ sufficiently small, the dimension of the kernel of $H$ is precisely $(\operatorname{deg} V)-1$.

Remark. This is a vanishing theorem for the following simple reason. Since $\Gamma$ commutes with $Q$, and therefore with $H$, we may decompose the Hilbert space $\mathscr{H}=\mathscr{H}_{+} \oplus \mathscr{H}_{-}$, the eigenspaces of $\Gamma$, and write

$$
\operatorname{dim} \operatorname{ker} H=\operatorname{dim} \operatorname{ker} H \Upsilon_{\mathscr{H}_{+}}+\operatorname{dim} \operatorname{ker} H \Upsilon_{\mathscr{H}_{-}} ;
$$

then considering (1.3) in the limit $\tau \rightarrow \infty$ we have

$$
\operatorname{ind}(Q)=\operatorname{dim} \operatorname{ker} H \uparrow_{\mathscr{H}_{+}}-\operatorname{dim} \operatorname{ker} H \uparrow_{\mathscr{H}_{-}},
$$

and Theorem 2 may be restated simply as

$$
\operatorname{dim} \operatorname{ker} H \uparrow_{\mathscr{H}_{-}}=0 .
$$

Thus Theorem 2 says that the kernel of an operator on a graded vector space lies wholly within a subspace of fixed grading. Our result is in this way reminiscent of the vanishing theorems appearing in algebraic geometry [5]. However, while in finite dimension such theorems can be proved using local bounds on the operators in question, we know of no such bounds for the quantum field theoretic operators of the Wess-Zumino model. Instead we use a different method, which relies on the exponential decay of the path integral representations of the heat kernel and index.

It is interesting to note that the vanishing theorem is not expected to hold for the $N=1$ Wess-Zumino models, where the index can only take the values \pm 1 and 0 . The $N=2$ Wess-Zumino models differ from those in that there is a natural 
complex structure on the Hilbert space in question, which respects the structure of the operators $H$ and $Q$. This is reminiscent of the finite dimensional situation, where vanishing theorems occur naturally in the holomorphic setting. It is curious that our methods do not make direct use of the complex structure. The consequences of this structure enter in the construction of the cluster expansion; the technical differences with the $N=1$ case are discussed in [7].

Proof of Theorem 2. We have

and

$$
\operatorname{ind}(Q)=\operatorname{Tr} \Gamma e^{-\tau H}
$$

$$
\operatorname{dim} \operatorname{ker} H=\lim _{\tau \rightarrow \infty} \operatorname{Tr} e^{-\tau H} .
$$

Since $Q$ is selfadjoint, $H=Q^{2} \geqq 0$; combined with $e^{-\tau H}$ being trace class, we have, for $\tau$ sufficiently large,

$$
\operatorname{dim} \operatorname{ker} H=\operatorname{Tr} e^{-\tau H}+O\left(e^{-\tau \varepsilon}\right),
$$

for some $\varepsilon>0$. But by Theorem 3, we have, for $\tau$ sufficiently large,

$$
\left|\operatorname{Tr} \Gamma e^{-\tau H}-\operatorname{Tr} e^{-\tau H}\right|<1 / 2 .
$$

Thus

$$
|\operatorname{dim} \operatorname{ker} H-\operatorname{ind}(Q)|<1 ;
$$

since $\operatorname{dim} \operatorname{ker} H$ and ind $(Q)$ are integers, they must be equal.

The main technical result needed to prove this theorem, and the technical core of this paper, is the following estimate.

Theorem 3. For $\lambda$ and $\xi$ sufficiently small, for every l there exists $\tau_{l}>0$ such that for $\tau>\tau_{l}$,

$$
\left|\operatorname{Tr} e^{-\tau H}-\operatorname{Tr} \Gamma e^{-\tau H}\right|<1 / 2 .
$$

To prove this theorem we will make use of the Euclidean methods of $[8,9]$. In [8] it was shown that the trace and graded trace of the heat kernel of $H$ could be expressed in terms of integrals of certain functions $\mathscr{F}_{a}, \mathscr{F}_{p}$ on the space $\mathscr{S}^{\prime}\left(T_{l} \times T_{\tau}\right)$ of distributions on the torus, with respect to the Gaussian measure $d \mu_{\mathrm{c}}$ with periodic covariance on the torus $T_{l} \times T_{\tau}$. The functions $\mathscr{F}_{a}$ and $\mathscr{F}_{p}$ differ in the boundary conditions (antiperiodic $v$ s. periodic in time) imposed on a Fredholm determinant appearing in each of them.

To deal with this difference we apply the cluster expansion methods of [9], which allow us to control the dependence of such integrals on the boundary conditions. In [9], we introduced an integer lattice on the torus $T_{l} \times T_{\tau}$, and interpolated between the covariance $C$ and the covariance $C_{D}$ with Dirichlet boundary conditions on each bond of this lattice. We also interpolated between the functions $\mathscr{F}_{a}, \mathscr{F}_{p}$ and a similar function $\mathscr{F}_{D}$ which is a product of functions, each depending on the value of a distribution within each block of the lattice. We then applied the fundamental theorem of calculus, in the usual form for cluster expansions [6], to obtain the following result.

$$
\begin{aligned}
& \text { Let } \Xi(l, \tau)=\prod_{k=1}^{\infty} \operatorname{coth}[\tau \omega(2 \pi k / l)] \text {, where } \omega(x)=\left(x^{2}+m^{2}\right)^{1 / 2} \text {. [For fixed } l \text {, } \\
& \Xi(l, \tau) \rightarrow 1 \text { as } \tau \rightarrow \infty .]
\end{aligned}
$$


Theorem 4 [9]. For $\lambda$ and $\xi$ sufficiently small, the trace (respectively graded trace) of the heat kernel of $H$ is given by a cluster expansion.

$$
\begin{gathered}
\operatorname{Tr} e^{-\tau H}=\Xi(l, \tau) \sum_{n=0}^{\infty} \frac{1}{n !} \sum_{\substack{\left\{\Gamma_{1}, \ldots, \Gamma_{n}\right\} \\
\text { compatible }}} \varrho_{a}\left(\Gamma_{1}\right) \ldots \varrho_{a}\left(\Gamma_{n}\right), \\
\operatorname{Tr} \Gamma e^{-\tau H}=\sum_{n=0}^{\infty} \frac{1}{n !} \sum_{\substack{\left\{\Gamma_{1}, \ldots, \Gamma_{n}\right\} \\
\text { compatible }}} \varrho_{p}\left(\Gamma_{1}\right) \ldots \varrho_{p}\left(\Gamma_{n}\right),
\end{gathered}
$$

where the activities $\varrho_{a}, \varrho_{p}$ satisfy the estimate

$$
\left|\varrho_{\#}(\Gamma)\right|<\exp (-c|\Gamma|),
$$

where $\Gamma \subset T_{e} \times T_{\tau}$. The free energy of each cluster expansion is zero.

The basic idea of the proof of Theorem 3 is as follows. We use Theorem 4 to write the difference between the trace and graded trace of the heat kernel as a sum of terms, each involving a product of factors $\varrho_{p}(\Gamma)$ and at least one difference factor $\varrho_{a}(\Gamma)-\varrho_{p}(\Gamma)$. We will then show that each such difference factor satisfies an estimate of the form (1.17), with an additional small factor $e^{-c \tau}$, where $c$ is some constant; the resummation of the remaining terms will introduce an error of order $\exp \left[O\left(l \tau e^{-l}\right)\right]$, due to vanishing of the infinite volume free energy. Thus

$$
\left|\operatorname{Tr} \Gamma e^{-\tau H}-\operatorname{Tr} e^{-\tau H}\right| \leqq \exp \left[-c \tau+O\left(l \tau e^{-l}\right)\right],
$$

so that, choosing $l$ and $\tau$ sufficiently large, we obtain Theorem 3 .

\section{Cluster Expansions for $N=2$ Wess-Zumino Models}

In this section we recall the cluster expansion developed in $[7,9]$ for the $N=2$ Wess-Zumino models. These results will be used in Sect. 3 to arrive at the proof of our main estimate in Theorem 3.

Let $\mathscr{S}^{\prime}\left(T_{l} \times T_{\tau}\right)$ denote the space of tempered distributions on the torus $T_{l} \times T_{\tau}$. On $\mathscr{S}^{\prime}\left(T_{l} \times T_{\tau}\right)$ there is defined the Gaussian measure $d \mu_{C}$ with covariance

$$
C(x, y)=\sum_{p \in \frac{2 \pi}{l} \mathbb{Z} \times \frac{2 \pi}{\tau} \mathbb{Z}} \frac{e^{i p \cdot(x-y)}}{p^{2}+m^{2}} .
$$

Similarly, let $S_{p}(x, y), S_{a}(x, y)$ denote the Fermionic covariance with periodic boundary conditions on $T_{l}$ and periodic (for $S_{p}$ ) or antiperiodic (for $S_{a}$ ) boundary conditions on $T_{\tau}$, defined on the Hilbert space $\mathscr{H}=\mathscr{H}_{-1 / 2}\left(\mathbb{R}^{2}\right) \oplus \mathscr{H}_{-1 / 2}\left(\mathbb{R}^{2}\right)$ :

$$
\begin{gathered}
S_{p}(x, y)=\sum_{\substack{p_{1} \in \frac{2 \pi}{l} \mathbb{Z} \\
p_{2} \in \frac{2 \pi}{\tau} \mathbb{Z}}} \gamma_{0} \frac{-\not p+m}{p^{2}+m^{2}} e^{i p \cdot(x-y)} \\
S_{a}(x, y)=\sum_{\substack{p_{1} \in \frac{2 \pi}{l} \mathbb{Z} \\
p_{2} \in \frac{\pi}{\tau}(2 \mathbb{Z}+1)}} \gamma_{0} \frac{-\not p+m}{p^{2}+m^{2}} e^{i p \cdot(x-y)} .
\end{gathered}
$$

Here we write $\not p=\gamma_{0}^{E} p_{2}+\gamma_{1}^{E} p_{1}$ with

$$
-i \gamma_{0}=\gamma_{0}^{E}=\left[\begin{array}{rr}
0 & -1 \\
1 & 0
\end{array}\right], \quad \gamma_{1}^{E}=\left[\begin{array}{ll}
0 & i \\
i & 0
\end{array}\right] \text {. }
$$


We may then define the Fredholm determinant

$$
\operatorname{det}_{3}\left[1+K_{\sharp}(\phi)\right] \text {, }
$$

where

$$
K_{\sharp}(\phi)=S_{\sharp} \gamma_{0} \chi_{\Lambda}\left(\Upsilon\left(V^{\prime \prime}(\phi)\right)-m\right)
$$

and

$$
\Upsilon(z) \equiv\left[\begin{array}{cc}
z & 0 \\
0 & z^{*}
\end{array}\right]
$$

and the functionals

$$
\begin{gathered}
\tilde{\mathscr{A}}_{\sharp}(\phi)=R_{\sharp}(\phi)+\int_{A}\left(:\left|V^{\prime}(\phi)\right|^{2}:-m^{2}:|\phi|^{2}:\right) d x, \\
\mathscr{F}_{\sharp}(\phi)=\exp -\tilde{\mathscr{A}}_{\sharp}(\phi) \cdot \operatorname{det}_{3}\left[1+K_{\sharp}(\phi)\right],
\end{gathered}
$$

where

$$
R_{\sharp}(\phi)=\int_{A} d x\left[|V(\phi)|^{2}-:|V(\phi)|^{2}:-m^{2}|\phi|^{2}+m^{2}:|\phi|^{2}:\right]+\frac{1}{2} \operatorname{Tr} K_{\sharp}^{2}(\phi)-\operatorname{Tr} K_{\sharp}(\phi) .
$$

In [8] it is shown that $\mathscr{F}_{\sharp}(\phi)$ is well defined [a.e. $\left(d \mu_{c}\right)$ ] as a limit of regularized expressions, and that it is integrable with respect to $d \mu_{c}(\phi)$.

To develop a cluster expansion we define the decoupled versions of all these functions. We introduce a lattice $L \mathbb{Z}^{2}$ of size $L$ in $T_{l} \times T_{\tau}$, and will interpolate between our original operators and decoupled operators which are designed so that the fully decoupled partition functions factor into a product over all the squares of this lattice. This procedure is lengthy but straightforward, and may be found in Sect. 3 of [9]; here we will consider a "condensed version" where we neglect features not essential to our current analysis.

To do this we first note that

$$
\operatorname{det}_{3}[1+S \zeta] \Gamma_{\mathscr{H}}=\operatorname{det}_{3}\left[1+D^{1 / 2} S \zeta D^{-1 / 2}\right] \Gamma_{\mathscr{C}},
$$

where $\zeta$ is a function of the scalar field (cf. (3.6) of [9]), $\mathscr{H}^{\prime}=L^{2}\left(\mathbb{R}^{2}\right) \oplus L^{2}\left(\mathbb{R}^{2}\right)$, and $D=\left(-\Delta+m^{2}\right)^{1 / 2}$. We write

$$
D^{1 / 2} S \zeta D^{-1 / 2}=\mathscr{U} D^{-1 / 2} \zeta D^{-1 / 2},
$$

where $\mathscr{U}=D^{1 / 2} S D^{1 / 2}$, and decouple this operator by writing

$$
K(s)=(\mathscr{U})_{s}\left(D^{-1 / 2}\right)_{s} \zeta\left(D^{-1 / 2}\right)_{s},
$$

where

$$
\mathcal{O}_{s} \equiv \sum_{\Delta} \chi_{\Delta} \mathcal{O} \chi_{\Delta}+\sum_{\Delta \neq \Delta^{\prime}} H\left(s, \Delta, \Delta^{\prime}\right) \chi_{\Delta} \mathcal{O} \chi_{\Delta^{\prime}},
$$

and $0 \leqq H\left(s, \Delta, \Delta^{\prime}\right) \leqq 1$ is a function indicating the degree of decoupling between $\Delta$ and $\Delta^{\prime}$; see (3.28-29) of [9] or (II.10-13) of [1]. Most importantly $H\left(s, \Delta, \Delta^{\prime}\right)=0$ if there is no path from $\Delta$ to $\Delta^{\prime}$ that does not cross a bond with $s_{b}=0$, so that $\exp \mathcal{O}_{s}$ will factor over regions whose boundaries are bonds with $s_{b}=0$.

In the case of periodic or antiperiodic covariance, our Fermionic kernels are

$$
\begin{gathered}
K_{p}(s)=\left(\sum_{u \in \mathbb{Z}^{2}} D^{1 / 2} S D^{1 / 2} T_{u(l, \tau)}\right)_{s}\left(D^{-1 / 2}\right)_{s} \zeta\left(D^{-1 / 2}\right)_{s}, \\
K_{a}(s)=\left(\sum_{u \in \mathbb{Z}^{2}}(-1)^{u_{2}} D^{1 / 2} S D^{1 / 2} T_{u(l, \tau)}\right)_{s}\left(D^{-1 / 2}\right)_{s} \zeta\left(D^{-1 / 2}\right)_{s},
\end{gathered}
$$


where $T_{u}: \mathscr{H}^{\prime} \rightarrow \mathscr{H}^{\prime}$ is the (unitary) translation operator: $\left(T_{u} f\right)(x)=f(x+u)$, and $u(l, \tau)=u_{1} \vec{l}+u_{2} \vec{\tau}$.

For the Boson we consider a partially decoupled measure $d \mu_{s}(\phi)$ with covariance $C_{s}$; it is convenient to use replacement operator notation [4] so that

$$
\partial_{s_{b}} \int d \mu_{s} G=\int d \mu_{s} \int d x d y\left(\frac{\partial C_{s}}{\partial s_{b}}\right)(x, y) \frac{\delta^{2}}{\delta \phi^{*}(x) \delta \phi(y)} G
$$

and

$$
\int d x d y\left(\partial^{\gamma} C_{s}\right)(x, y) \frac{\delta^{2}}{\delta \phi^{*}(x) \delta \phi(y)} G=\mathscr{E}_{\gamma} r_{\gamma}^{*} r_{\gamma} G .
$$

The G-J-S cluster expansion [6] then takes the following form [9], where $Z_{\sharp}(s)$ is obtained from $\int \mathscr{F}_{\sharp}(\phi) d \mu(\phi)$ by replacing $K_{\sharp}(\phi)$ by $K_{\sharp}(s)$ (and $\tilde{\mathscr{A}}_{\sharp}$ by $\mathscr{A}_{\sharp}$; corrections arise in replacing $\gamma_{0} \chi_{A}\left(Y\left(V^{\prime \prime}(\phi)\right)-m\right)$ by $\zeta$ in the determinant [7]):

\section{Proposition 2.1.}

$$
Z_{\sharp}(\Lambda)=\sum_{\Gamma} \int d s_{\Gamma} \partial^{\Gamma} Z_{\sharp}(s) .
$$

Note. Our cluster activities $\varrho(\Gamma)$ are essentially given by $\int d s_{\Gamma} \partial^{\Gamma} Z_{\sharp}(s)$; see (3.62-65) of [9].

Next we sketch a formula for these derivatives; details are in [9].

Let $\pi$ be a partition of $\Gamma$. As in [4], decompose this as

$$
\begin{gathered}
\pi=\pi_{b} \cup \pi_{f}, \\
\pi_{f}=\pi_{f, A} \cup \pi_{f, E} \cup \pi_{f, B} \cup \pi_{f, s} \cup \pi_{f, 0}, \\
\pi_{b} \cup \pi_{b}^{*}=\pi_{b, A} \cup \pi_{b, E} \cup \pi_{b, B} \cup \pi_{b, s} \cup \pi_{b, 0} .
\end{gathered}
$$

Then we have

Lemma 2.2. We can write $\partial^{\Gamma} Z_{\sharp}(s)$ according to the following decomposition:

$$
\begin{aligned}
\partial^{\Gamma} Z_{\sharp}(s)= & \sum_{\pi \in \mathscr{P}(\Gamma)} \sum_{\text {decompositions }} \int d \mu_{s}(\phi) e^{-\mathscr{A}_{\sharp}} \mathscr{E}\left(\pi_{b}\right) \\
& \times\left[\partial^{\pi_{f, s}} r\left(\pi_{b, S}\right) B\left(\pi_{f, B}, \pi_{b, B}\right) \partial^{\pi_{f, 0}} r\left(\pi_{b, 0}\right) \tau_{r}\left(A\left(\pi_{f, A}, \pi_{b, A}\right) \cdot d \wedge^{r} E\left(\pi_{f, E}, \pi_{b, E}\right)\right)\right],
\end{aligned}
$$

with the notation explained below.

We have determinant minors of the form

$$
\tau_{k}(G) \equiv k ! \operatorname{Tr}\left(\bigwedge^{k}[1+K(s)]^{-1} \cdot G\right) \operatorname{det}_{3}[1+K(s)],
$$

and the shorthand replacement notation

$$
r\left(\pi_{b, X}\right)=\prod_{\gamma \in \pi_{b, X}} r_{\gamma} \prod_{\gamma^{*} \in \pi_{b, X}} r_{\gamma^{*}}^{*} .
$$

We also have

$$
\begin{gathered}
B\left(\pi_{f, B}, \pi_{b, B}\right)=\prod_{\gamma \in \pi_{f}, B}\left(-\partial_{s}^{\gamma} \mathscr{A}_{\sharp}\right) \prod_{\gamma \in \pi_{b}, \boldsymbol{B}}\left(-r_{\gamma^{\prime}} \mathscr{A}_{\sharp}\right) \prod_{\gamma^{*} \in \pi_{b, B}}\left(-r_{\gamma^{*}}^{*} \mathscr{A}_{\sharp}\right), \\
A\left(\pi_{f, A}, \pi_{b, A}\right)=\bigwedge_{\gamma \in \pi_{f}, A} K_{\sharp}^{2}(s) \partial_{s}^{\gamma} K_{\sharp}(s) \bigwedge_{\gamma \in \pi_{b, A}} K_{\sharp}^{2}(s) r_{\gamma} K_{\sharp}(s) \bigwedge_{\gamma^{*} \in \pi_{b, A}} K_{\sharp}^{2}(s) r_{\gamma^{*}}^{*} K_{\sharp}(s)
\end{gathered}
$$


and

$$
\begin{aligned}
d \wedge^{r} E\left(\pi_{f, E}, \pi_{b, E}\right)= & \prod_{\gamma \in \pi_{f, E}} d^{\prime} \wedge^{r}\left(1-K_{\#}(s)\right) \partial_{s}^{\gamma} K_{\#}(s) \prod_{\gamma \in \pi_{b, E}} d^{\prime} \wedge^{r}\left(1-K_{\#}(s)\right) r_{\gamma} K_{\#}(s) \\
& \times \prod_{\gamma^{*} \in \pi_{b, E}} d^{\prime} \wedge^{r}\left(1-K_{\#}(s)\right) r_{\gamma^{*}}^{*} K_{\#}(s),
\end{aligned}
$$

where $r=\left|\pi_{f, A}\right|+\left|\pi_{b, A}\right|$ and the $d^{\prime} \wedge$ means that terms where $E$ derivatives precede $A$ derivatives (according to an arbitrary ordering) are omitted. Finally, we have the convention that $\pi_{f, s}, \pi_{b, s}, \pi_{f, 0}$, and $\pi_{b, 0}$ derivatives only act on already differentiated terms, one term per derivative.

The terms in (2.16) are estimated by fixing all the localization squares in the sum over characteristic functions within the decoupling. We indicate such localized terms by writing $A_{l}, B_{l}$, etc., and rewrite Eq. (2.16) to make this explicit:

Lemma 2.3. The derivative $\partial^{\Gamma} Z_{\sharp}(s)$ may be written as the following sum over localized terms:

$$
\begin{aligned}
\partial^{\Gamma} Z_{\sharp}(s)= & \sum_{\pi \in \mathscr{P}(\Gamma)} \sum_{\begin{array}{c}
\text { decomps } \\
\text { localizations }
\end{array}} \int d \mu_{s}(\phi) e^{-\mathscr{A}_{\sharp}} \mathscr{E}\left(\pi_{b}\right) \\
& \times\left[\partial^{\pi_{f}, s_{r}} r\left(\pi_{b, S}\right) B_{l}\left(\pi_{f, B}, \pi_{b, B}\right) \partial^{\pi_{f}, 0} r\left(\pi_{b, 0}\right) \tau_{r}\left(A_{l}\left(\pi_{f, A}, \pi_{b, A}\right) \cdot d \wedge^{r} E_{l}\left(\pi_{f, E}, \pi_{b, E}\right)\right)\right] .
\end{aligned}
$$

When we combine the estimates on all these localized terms we arrive at the exponentially decaying bound (1.17).

\section{Small Factors}

In this section we show how to compare the cluster expansions for $Z_{p}$ and $Z_{a}$. We will show that the difference of the two is a sum of terms (in the form of a cluster expansion), each of which contains a small factor. The summation of these terms is left to the next section.

By subtracting the periodic cluster expansion (1.16) from the anti-periodic cluster expansion $[(1.15)$ without the prefactor $]$ we have

$$
\begin{aligned}
Z_{a}-Z_{p}= & \sum_{n=0}^{\infty} \frac{1}{n !} \sum_{\substack{\left\{\Gamma_{1}, \ldots, \Gamma_{n}\right\} \\
\text { compatible }}} \sum_{k \leqq n} \varrho_{p}\left(\Gamma_{1}\right) \ldots \varrho_{p}\left(\Gamma_{k-1}\right)\left(\varrho_{a}\left(\Gamma_{k}\right)-\varrho_{p}\left(\Gamma_{k}\right)\right) \\
& \times \varrho_{a}\left(\Gamma_{k+1}\right) \ldots \varrho_{a}\left(\Gamma_{n}\right) \\
= & \sum_{\substack{n=1 \\
m=0}}^{\infty} \frac{1}{n ! m !} \sum_{\substack{\left\{\Gamma_{1}, \ldots, \Gamma_{n+m}\right\} \\
\text { compatible }}} \varrho_{d}\left(\Gamma_{1}\right) \ldots \varrho_{d}\left(\Gamma_{n}\right) \varrho_{p}\left(\Gamma_{n+1}\right) \ldots \varrho_{p}\left(\Gamma_{n+m}\right),
\end{aligned}
$$

where

$$
\varrho_{d}(\Gamma)=\varrho_{a}(\Gamma)-\varrho_{p}(\Gamma)
$$

We wish to prove the following result:

Proposition 3.1. For $\lambda$ and $\xi$ sufficiently small, if $\tau$ is sufficiently large the difference $Z_{a}-Z_{p}$ satisfies

$$
\left|Z_{a}-Z_{p}\right|<1 / 4
$$




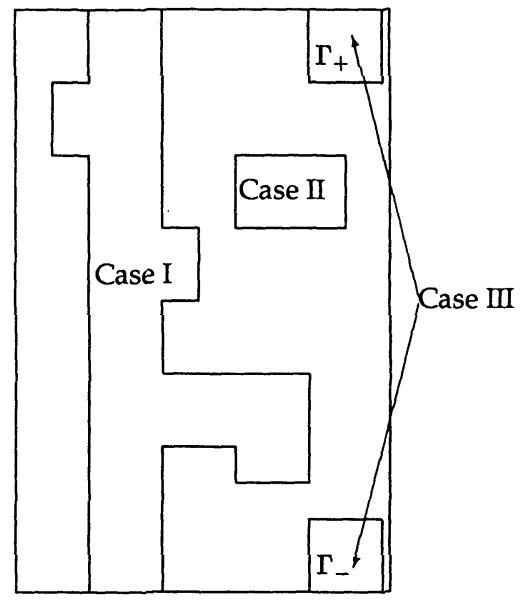

Fig. 1. The $l \times \tau$ region and three clusters illustrating the three cases of Lemma 3.2

The first step is the following lemma:

Lemma 3.2. The factor $\varrho_{a}(\Gamma)-\varrho_{p}(\Gamma)$ satisfies

$$
\left|\varrho_{a}(\Gamma)-\varrho_{p}(\Gamma)\right| \leqq \exp \left[-c_{1}|\Gamma|-c_{2} \tau\right]
$$

where $c_{1}, c_{2}>0$.

Proof. There are three cases (see Fig. 1).

Case $I$. $\Gamma$ 's length is $O(\tau)$ (this allows wrapping around the strip).

In this case, Theorem 4 guarantees that for some $c>0$,

$$
\left|\varrho_{a}(\Gamma)\right|,\left|\varrho_{p}(\Gamma)\right|<e^{-c|\Gamma|} .
$$

Since $|\Gamma| \gtrsim \tau$, we have

$$
\left|\varrho_{a}(\Gamma)-\varrho_{p}(\Gamma)\right| \leqq \exp [-c|\Gamma| / 2-c \tau / 2],
$$

as needed.

Case II. $\Gamma$ does not cross the top of the strip, and its length is less than $O(\tau)$.

In this case we compare $\varrho_{p}(\Gamma)$ and $\varrho_{a}(\Gamma)$ by interpolating between the two different cluster activities:

$$
\varrho_{p}(\Gamma)-\varrho_{a}(\Gamma)=\int_{0}^{1} d t \frac{\partial}{\partial t} \varrho_{t}(\Gamma),
$$

where we denote by $\varrho_{t}(\Gamma)$ the cluster activity corresponding to $\Gamma$ in the cluster expansion with a linear combination of boundary conditions [we will identify the specific nature of the interpolation later - cf. (3.8)]. Then

$$
\left|\varrho_{p}(\Gamma)-\varrho_{a}(\Gamma)\right| \leqq \sup _{t}\left|\frac{\partial \varrho_{t}(\Gamma)}{\partial t}\right| .
$$


Recall, however, the form of the activity $\varrho_{t}(\Gamma)$. It must be a sum of terms of the form

$$
\begin{aligned}
& \int d \mu_{s}(\phi) e^{-\mathscr{A}_{t}} \mathscr{E}\left(\pi_{b}\right)\left[\partial^{\pi_{f, s}} r\left(\pi_{b, S}\right) B_{l}\left(\pi_{f, B}, \pi_{b, B}\right) \partial^{\pi_{f,}} r\left(\pi_{b, 0}\right)\right. \\
& \left.\quad \times \tau_{r}\left(A_{l}\left(\pi_{f, A}, \pi_{b, A}\right) \cdot d \wedge^{r} E_{l}\left(\pi_{f, E}, \pi_{b, E}\right)\right)\right],
\end{aligned}
$$

as in (2.22). In Lemma 7.2 of [9] we showed that such sums could be bounded in terms of the cluster $\Gamma$. An essential ingredient in the proof of that lemma was the exponential decay between the localization squares introduced in the cluster expansion. We wish to exploit this decay, so we "leave in" some of it.

Lemma 3.3 (Alternate version of Lemma 7.2 of [9]). For $\lambda$, $\xi$ sufficiently small, there exists $\delta>0$ such that

$$
\left|\partial^{\Gamma} Z_{t}(s)\right| \leqq G(\Gamma) \sup _{\substack{\text { decomps } \\ \text { localizations }}} \prod_{\substack{\text { localization } \\ \text { square pairs }}} e^{-\delta d\left(\Delta_{1}, \Delta_{2}\right)},
$$

where $G(\Gamma)$ can be summed to give the cluster bound (1.17).

Let us now consider the derivative $\partial \varrho / \partial t$. It is also given as a sum of the terms of the form (3.5), except that in addition to the s-derivatives defining the $A, E$, and $B$ terms one $t$-derivative must appear. We will show that this $t$-derivative gives rise to the additional factor $\exp [-O(\tau)]$.

Suppose, for example, the $t$-derivative appears in an $A$-type term. Recall that $A$-terms are of the form

$$
A=K_{t}^{2}(s) \partial_{s}^{\gamma} K_{t}(s),
$$

where the Fermionic kernel is

$$
K_{t}(s)=t K_{p}(s)+(1-t) K_{a}(s) .
$$

Thus

$$
\frac{\partial K_{t}(s)}{\partial t}=2\left(\sum_{u \in \mathbb{Z}^{2}, u_{2} \text { odd }}(-1)^{u_{2}} D^{1 / 2} S D^{1 / 2} T_{u(l, \tau)}\right)_{s}\left(D^{-1 / 2}\right)_{s} \zeta\left(D^{-1 / 2}\right)_{s} .
$$

Now observe that in the sum (3.9) the first term, corresponding to the value $u=(0,0)$, is missing. Thus the product over localization pairs in (3.6) will include at least one pair that is $O(\tau)$ apart. Summing over all the terms giving rise to the activity $\varrho$, we obtain an additional factor $\exp [-O(\tau)]$.

It is immediately clear that the same behavior is to be expected in when the $t$-derivative occurs in $E$ or $B$ terms; somewhere there will be a kernel $\partial K_{t}(s) / \partial t$ which will give rise to a sum of terms, in each of which a pair of localization squares are forced to be at least $\tau$ apart.

Case III. $\Gamma$ crosses the top of the strip, and its length is less than $O(\tau)$.

In this case we cannot directly use the formula (3.9), since the term corresponding to the first translate $\left(u_{2}= \pm 1\right)$ in (3.9) may not be small. To overcome this difficulty, we write

$$
\Gamma=\Gamma_{+} \cup \Gamma_{-},
$$

where $\Gamma_{+}$lies on one side of the edge, and $\Gamma_{-}$on the other. This is well-defined since $\Gamma$ does not wrap around the strip (see Fig. 1).

With this decomposition we have a similar decomposition of the Hilbert space,

$$
\mathscr{H}^{\prime}(\Gamma)=\mathscr{H}^{\prime}\left(\Gamma_{+}\right) \oplus \mathscr{H}^{\prime}\left(\Gamma_{-}\right) .
$$


We define the operator $\tilde{K}_{t}(s)$ on $\mathscr{H}^{\prime}(\Gamma)$ according to the following kernel which is partially "reversed" on the off-diagonal portion:

$$
\begin{aligned}
& \tilde{K}_{t}(s)(x, y) \\
& \quad= \begin{cases}K_{t}(s)(x, y), & x, y \in \Gamma_{+} \text {or } x, y \in \Gamma_{-}, \\
-t K_{p}(s)(x, y)+(1-t) K_{a}(s)(x, y), & x \in \Gamma_{+}, \quad y \in \Gamma_{-} \quad \text { or } \quad x \in \Gamma_{-}, \quad y \in \Gamma_{+} .\end{cases}
\end{aligned}
$$

For $t=0$ this is the same as $K_{t}(s)-$ see (3.8). For $t=1, \tilde{K}_{t}(s)$ is $K_{t}(s)$ on the block diagonal and $-K_{t}(s)$ off the block diagonal. Thus any trace of any product of $K_{t}(s)$ 's (or their derivatives) for $t=0$ or $t=1$ is unchanged when we replace $K_{t}(s)$ by $\tilde{K}_{t}(s)$ as any nonvanishing trace must have an even number of off diagonal terms; since the determinant (and its minors) can be represented as sums of traces, this holds for the determinant (and its minors) as well. Thus the difference $\varrho_{p}(\Gamma)-\varrho_{a}(\Gamma)$ may be written as an interpolation similar to (3.4), except that we replace $\varrho_{t}(\Gamma)$ by $\tilde{\varrho}_{t}(\Gamma)$ and $K_{t}(s)$ by $\widetilde{K}_{t}(s)$. We have

$$
\left|\varrho_{p}(\Gamma)-\varrho_{a}(\Gamma)\right| \leqq \sup _{t}\left|\frac{\partial \tilde{\varrho}_{t}(\Gamma)}{\partial t}\right|,
$$

where, again, we obtain a sum of terms with a term containing $\partial \tilde{K}_{t}(s) / \partial t$ appearing in an $A, E$ or $B$ term. However,

$$
\begin{aligned}
\frac{\partial \widetilde{K}_{t}(s)}{\partial t}= & 2 \sum_{\Gamma_{x}=\Gamma_{+}, \Gamma_{-}} \chi_{\Gamma_{x}}\left(\sum_{u \in \mathbb{Z}^{2}, u_{2} \text { odd }} D^{1 / 2} S D^{1 / 2} T_{u(l, \tau)}\right)_{s}\left(D^{-1 / 2}\right)_{s} \zeta\left(D^{-1 / 2}\right)_{s} \chi_{\Gamma_{x}} \\
& -2 \sum_{\substack{\Gamma_{x}=\Gamma_{+}, \Gamma_{y}=\Gamma_{-} ; \text {or } \\
\Gamma_{x}=\Gamma_{-}, \Gamma_{y}=\Gamma_{+}}} \chi_{\Gamma_{x}}\left(\sum_{u \in \mathbb{Z}^{2}, u_{2} \text { even }} D^{1 / 2} S D^{1 / 2} T_{u(l, \tau)}\right)_{s} \\
& \times\left(D^{-1 / 2}\right)_{s} \zeta\left(D^{-1 / 2}\right)_{s} \chi_{\Gamma_{y}} .
\end{aligned}
$$

The first term of (3.11) is now of the same form as (3.9), and so produces a factor $\exp [-O(\tau)]$. In the second term of (3.11) the restriction that $u_{2}$ be even prevents $\Gamma_{x}$ from being translated next to $\Gamma_{y}$ and we again obtain the decay $\exp [-O(\tau)]$.

\section{Resummation}

In this section we will complete the proof of Proposition 3.1 by combining the formula given in Eq. (3.1) for $Z_{a}-Z_{p}$ with the estimate of Lemma 3.2 for the difference of the activities $\varrho_{a}(\Gamma)-\varrho_{p}(\Gamma) \mid$. The crucial fact we still need is the vanishing of the free energy in each phase of the model, proved in [9] following the methods of [2].

Let us examine the sum appearing in (3.1). Each term is of the form

$$
\varrho_{d}\left(\Gamma_{1}\right) \ldots \varrho_{d}\left(\Gamma_{k}\right) \varrho_{p}\left(\Gamma_{k+1}\right) \ldots \varrho_{p}\left(\Gamma_{n}\right) .
$$

Our strategy will be to divide the clusters appearing on this sum into large and small clusters, and resum the small clusters.

Let $\Gamma$ be a large cluster if it is associated with a $\varrho_{d}$ factor, if $|\Gamma|>l$, or if it surrounds another large cluster. Thus all $\Gamma$ that wrap around the strip (in either the vertical or horizontal direction) are large. Small clusters are those that are not large. 
We rewrite the sum appearing in (3.1) as follows:

$$
\begin{aligned}
Z_{a}-Z_{p}= & \sum_{\substack{n=1, m=0}}^{\infty} \frac{1}{n ! m !} \sum_{\substack{\left\{\Gamma_{1}, \ldots, \Gamma_{n+m}\right\} \\
\text { large, compatible }}} \varrho_{d}\left(\Gamma_{1}\right) \ldots \varrho_{d}\left(\Gamma_{n}\right) \varrho_{p}\left(\Gamma_{n+1}\right) \ldots \varrho_{p}\left(\Gamma_{n+m}\right) \\
& +\sum_{q=0}^{\infty} \frac{1}{q !} \sum_{\substack{\left\{\Gamma_{1}^{\prime}, \ldots, \Gamma_{q}^{\prime}\right\} \text { small } \\
\text { compatible with }\left\{\Gamma_{1}, \ldots, \Gamma_{n+m}\right\}}} \varrho_{p}\left(\Gamma_{1}^{\prime}\right) \ldots \varrho_{p}\left(\Gamma_{q}^{\prime}\right) .
\end{aligned}
$$

We now fix $\Gamma_{1}, \ldots, \Gamma_{n+m}$. Let

$$
Z_{\text {small }}=\sum_{q=0}^{\infty} \frac{1}{q !} \sum_{\begin{array}{c}
\left\{\Gamma_{1}^{\prime}, \ldots, \Gamma_{q}^{\prime}\right\} \text { small, } \\
\text { compatible with }\left\{\Gamma_{1}, \ldots, \Gamma_{n+m}\right\}
\end{array}} \varrho_{p}\left(\Gamma_{1}^{\prime}\right) \ldots \varrho_{p}\left(\Gamma_{q}^{\prime}\right) .
$$

We prove the following lemma:

Lemma 4.1. For appropriate values of the parameters of $V$ and for $\tau$ sufficiently large,

$$
\left|Z_{\text {small }}\right| \leqq \exp \left[O\left(\left|\partial \Gamma_{1}\right|+\ldots+\left|\partial \Gamma_{n+m}\right|\right)+e^{-O(l)} l \tau\right]
$$

Proof. The clusters $\Gamma_{1}, \ldots, \Gamma_{n+m}$ divide the volume $\Lambda$ into a number of sub-volumes (say $\left\{\Lambda^{(i)}\right\}$ ) with fixed boundary conditions. Then we can write

$$
Z_{\text {small }}=\prod_{i} Z_{\text {small }}\left(\Lambda^{(i)}\right) \text {. }
$$

Now we make use of the results from $[2,9]$ that the free energy of each phase of the model is zero.

In the finite volume $\Lambda^{(i)}$, the complete (fixed boundary conditions) cluster expansion has zero free energy but may have a boundary contribution as large as

$$
\exp O\left(\left|\partial \Lambda^{(i)}\right|\right)
$$

The expansion for $Z_{\text {small }}\left(\Lambda^{(i)}\right)$ differs from this in that

- We have removed large clusters.

- We have periodic activities $\varrho_{p}$.

Both of the above alterations result in corrections of order $e^{-O(l)}$ to the free energy. Thus

$$
\left|Z_{\text {small }}\left(\Lambda^{(i)}\right)\right| \leqq \exp \left[O\left(\left|\partial \Lambda^{(i)}\right|\right)+e^{-O(l)}\left|\Lambda^{(i)}\right|\right] .
$$

Since $\sum_{i}\left|\partial \Lambda^{(i)}\right|=\sum_{i}\left|\partial \Gamma_{i}\right|$, and $\sum_{i}\left|\Lambda^{(i)}\right| \leqq|\Lambda|=l \tau$, our proof is complete.

We may now proceed to the proof of Proposition 3.1. We have

$$
\begin{aligned}
\left|Z_{p}-Z_{a}\right| \leqq & \sum_{\substack{n=1, m=0}}^{\infty} \frac{1}{n ! m !} \sum_{\substack{\left\{\Gamma_{1}, \ldots, \Gamma_{n+m}\right\} \\
\text { large, compatible }}}\left|\varrho_{d}\left(\Gamma_{1}\right) \ldots \varrho_{d}\left(\Gamma_{n}\right) \varrho_{p}\left(\Gamma_{n+1}\right) \ldots \varrho_{p}\left(\Gamma_{n+m}\right)\right| \\
& \times \exp \left[O\left(\left|\partial \Gamma_{1}\right|+\ldots+\left|\partial \Gamma_{n+m}\right|\right)+e^{-O(l)} \mid \tau\right] .
\end{aligned}
$$

Each factor $\varrho_{d}(\Gamma)$ contributes a factor of $e^{-O(\tau+|\Gamma|)}$ by Lemma 3.2; each factor $\varrho_{p}\left(\Gamma_{\text {large }}\right)$ either contributes a factor of $e^{-O(l+|\Gamma|)}$ or surrounds another large cluster, in which case we only get $e^{-O(|\Gamma|)}$, but the entropy is reduced since $\Gamma$ cannot get too 
far from the cluster it surrounds:

$$
\begin{aligned}
\sum_{p=0}^{\infty} \frac{1}{p !} \sum_{\substack{\left\{\Gamma_{1}^{\prime}, \ldots, \Gamma_{p}^{\prime}\right\} \text { surround } \\
\text { other large clusters }}} \prod_{i=1}^{p} e^{-O\left(\left|\Gamma_{i}^{\prime}\right|\right)} & \leqq \sum_{p=0}^{\infty} \frac{1}{p !} O(n+m-p)^{p} \\
& =\exp O\left(n+m^{\prime}\right),
\end{aligned}
$$

where $m^{\prime}$ is the number of geometrically large "large" clusters. Thus

$$
\begin{aligned}
& \left|Z_{p}-Z_{a}\right| \\
& \quad \leqq \sum_{n=1}^{\infty} \frac{1}{n !} \sum_{\left\{\Gamma_{1}, \ldots, \Gamma_{n}\right\}} \prod_{i=1}^{n} e^{-O\left(\tau+\left|\Gamma_{i}\right|-1\right)} \sum_{m^{\prime}=0}^{\infty} \frac{1}{m^{\prime} !} \sum_{\left\{\Gamma_{n+1}, \ldots, \Gamma_{\left.n+m^{\prime}\right\}}\right.} \prod_{i=n+1}^{n+m^{\prime}} e^{-O\left(l+\left|\Gamma_{i}\right|-1\right)} \\
& \quad \leqq e^{-O(\tau)} \tau \exp \left[e^{-O(l)} \tau\right]
\end{aligned}
$$

if we adjust the parameters of $V$ so that our decay rates are greater than the entropy growth. We choose $\tau$ sufficiently large, and we are done.

We are now ready to prove Theorem 3 . We have

$$
\begin{aligned}
\left|\operatorname{Tr} e^{-\tau H}-\operatorname{Tr} \Gamma e^{-\tau H}\right| & =\left|Z_{p}-\Xi(l, \tau) Z_{a}\right| \\
& \leqq\left|Z_{p}-Z_{a}\right|+\left|Z_{a}\right| \cdot|1-\Xi(l, \tau)| \\
& \leqq\left|Z_{p}-Z_{a}\right|+\left|Z_{p}-Z_{a}\right| \cdot|1-\Xi(l, \tau)|+\left|Z_{p}\right| \cdot|1-\Xi(l, \tau)| .
\end{aligned}
$$

We showed earlier that $\left|Z_{p}-Z_{a}\right|<1 / 4$, and we have $Z_{p}=n-1$. Finally,

$$
|1-\Xi(l, \tau)|=O\left(e^{-\tau / l}\right)
$$

for large $\tau$, so that by taking $\tau \gg l$, we have

$$
\left|\operatorname{Tr} e^{-\tau H}-\operatorname{Tr} \Gamma e^{-\tau H}\right|<1 / 2
$$

as needed.

Acknowledgements. We would like to thank Arthur Jaffe and Konrad Osterwalder for helpful discussions. Additionally, after completing this work we learned of similar results by Christian Borgs and John Imbrie [3].

\section{References}

1. Balaban, T., Gawędzki, K.: A low temperature expansion for the pseudoscalar Yukawa theory of quantum fields in two spacetime dimensions. Ann. Inst. H. Poincaré, Section A 36, 271-400 (1982)

2. Borgs, C., Imbrie, J.Z.: A unified approach to phase diagrams in field theory and statistical mechanics. Commun. Math. Phys. 123, 305-328 (1989)

3. Borgs, C., Imbrie, J.Z.: Finite size scaling and surface tension from one dimension systems. Commun. Math. Phys. (to appear)

4. Cooper, A., Rosen, L.: The weakly coupled Yukawa ${ }_{2}$ field theory: cluster expansion and Wightman axioms. Trans. Am. Math. Soc. 234, 1-88 (1977)

5. Griffiths, P., Harris, J.: Principles of algebraic geometry. New York: Wiley 1978

6. Glimm, J., Jaffe, A., Spencer, T.: The particle structure of the weakly coupled $P(\phi)_{2}$ model and other applications of high temperature expansions. Part I: Physics of quantum field models. Part II: The cluster expansion. In: Constructive quantum field theory (Erice 1973). Velo, G., Wightman, A.S. (eds.). Berlin, Heidelberg, New York: Springer 1973 
Glimm, J., Jaffe, A., Spencer, T.: The Wightman axioms and particle structure in the $\mathscr{P}(\phi)_{2}$ quantum field model. Ann. Math. 100, 585-632 (1974)

Glimm, J., Jaffe, A., Spencer, T.: A convergent expansion about mean field theory. Ann. Phys. 101, 610-669 (1976)

7. Imbrie, J.Z., Janowsky, S.A., Weitsman, J.: Space-dependent Dirac operators and effective quantum field theory for fermions. Commun. Math. Phys. 135, 421-441 (1991)

8. Jaffe, A., Lesniewski: A priori estimates for $N=2$ Wess-Zumino models on a cylinder. Commun. Math. Phys. 114, 553-575 (1988)

Jaffe, A., Lesniewski, A., Weitsman, J.: Index of a family of Dirac operators on loop space. Commun. Math. Phys. 112, 75-88 (1987)

Jaffe, A., Lesniewski, A., Weitsman, J.: The two-dimensional, $N=2$ Wess-Zumino models on a cylinder. Commun. Math. Phys. 114, 147-165 (1988)

Jaffe, A., Lesniewski, A., Weitsman, J.: The loop space $S^{1} \rightarrow \mathbb{R}$ and supersymmetric quantum fields. Ann. Phys. 183, 337-351 (1988)

9. Janowsky, S.A.: The phase structure of the two-dimensional $Z=2$ Wess-Zumino model. Harvard University thesis (1990)

Janowsky, S.A., Weitsman, J.: The phase structure of the two-dimensional $N=2$ Wess-Zumino model. Commun. Math. Phys., to appear, Harvard University preprint HUTMP 90/B273 (1990)

Communicated by A. Jaffe 
